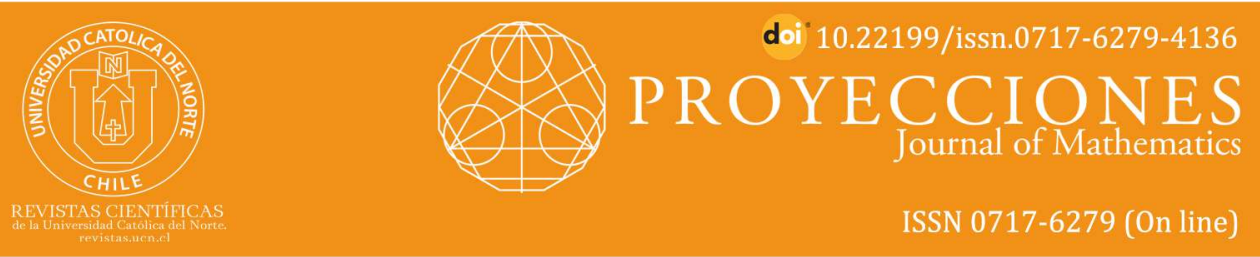

\title{
Ostrowski and Simpson type inequalities for multiplicative integrals
}

Muhammad Aamir Ali ${ }^{1}$ orcid.org/0000-0001-5341-4926

Hüseyin Budak ${ }^{2}$ (o orcid.org/0000-0001-8843-955X

Mehmet Zeki Sarikaya ${ }^{3}$ orcid.org/0000-0002-6165-9242

Zhiyue Zhang ${ }^{4}$ orcid.org/0000-0001-7070-2532

Nanjing Normal University, Jiangsu Key Lab. of NSLSCS, School of Mathematical Sci., Nanjing, China.

1⿴mahrmuhammad.aamir@gmail.com; 05298@njnu.cn

Düzce University, Dept. of Mathematics, Faculty of Science and Arts, Düzce, Turkey.

2匹hsyn.budak@gmail.com; ${ }^{3 \varpi}$ sarikayamz@gmail.com

Received: 30 April 2020 | Accepted: 23 December 2020

\section{Abstract:}

In this paper, we firstly obtain two identities for multiplicative differentiable functions. Then by using these identities, we establish Ostrowski and Simpson type inequalities for multiplicative integrals. At the end we give detail applications of our main results.

Keywords: Ostrowski inequality; Simpson inequality; Multiplicatively convex function; Multiplicative calculus; Integral inequalities.

MSC (2020): 26D15, 26B25, 26D10.

\section{Cite this article as (IEEE citation style):}

M. A. Ali, H. Budak, M. Z. Sarikaya, and Z. Zhang, "Ostrowski and Simpson type inequalities for multiplicative integrals ", Proyecciones (Antofagasta, On line), vol. 40, no. 3, pp. 743-763, 2021, doi: 10.22199/issn.0717-6279-4136

Article copyright: (C) 2021 Muhammad Aamir Ali, Hüseyin Budak, Mehmet Zeki Sarikaya, and Zhiyue Zhang. This is an open access article distributed under the terms of the Creative Commons License, which permits unrestricted use and distribution provided the original author and source are credited. 


\section{Introduction}

The study of various types of integral inequalities has been the focus of great attention for well over a century by a number of mathematicians, interested both in pure and applied mathematics. One of the many fundamental mathematical discoveries of A. M. Ostrowski is the following classical integral inequality associated with the differentiable mappings:

Theorem 1. [19] Let $f:[a, b] \rightarrow \mathbf{R}$ be a differentiable mapping on $(a, b)$ whose derivative $f^{\prime}:(a, b) \rightarrow \mathbf{R}$ is bounded on $(a, b)$, i.e. $\left\|f^{\prime}\right\|_{\infty}=$ $\sup \left|f^{\prime}(t)\right|<+\infty$. Then, we have the following inequality: $t \in(a, b)$

$$
\left|f(x)-\frac{1}{b-a} \int_{a}^{b} f(t) d t\right| \leq\left[\frac{1}{4}+\frac{\left(x-\frac{a+b}{2}\right)^{2}}{(b-a)^{2}}\right](b-a)\left\|f^{\prime}\right\|_{\infty},
$$

for all $x \in[a, b]$. The constant $\frac{1}{4}$ is the best possible.

One of the first generalization of Ostrowski's inequality was given in 1976 by Milovanović and Pečarić:

Theorem 2. [17] Let $f:[a, b] \rightarrow \mathbf{R}$ be twice differentiable function on $(a, b)$ whose derivative $f^{\prime \prime}:(a, b) \rightarrow \mathbf{R}$ is bounded on $(a, b)$, i.e. $\left\|f^{\prime \prime}\right\|_{\infty}=$ $\sup \left|f^{\prime \prime}(t)\right|<+\infty$. Then, we have the following inequality: $t \in(a, b)$

$$
\begin{array}{r}
\left|\frac{1}{2}\left(f(x)+\frac{(x-a) f(a)+(b-x) f(b)}{b-a}\right)-\frac{1}{b-a} \int_{a}^{b} f(t) d t\right| \\
\leq\left[\frac{1}{12}+\frac{\left(x-\frac{a+b}{2}\right)^{2}}{(b-a)^{2}}\right] \frac{(b-a)^{2}}{4}\left\|f^{\prime \prime}\right\|_{\infty}
\end{array}
$$

for all $x \in[a, b]$.

This result is a special case of a general result stated in [17] for $n$-times $(n>1)$ differentiable function with bounded $n$-derivative on $(a, b)$. The inequality (1.1) is a composite trapezoidal inequality, because

$$
\frac{b-a}{2}\left(f(x)+\frac{(x-a) f(a)+(b-x) f(b)}{b-a}\right)=\frac{x-a}{2}(f(a)+f(x))+\frac{b-x}{2}(f(x)+f(b))
$$


represents sum of two elementary trapezoidal formulas. For $x=(a+$ $b) / 2$ it reduces to a symmetric composite trapezoidal formula, and the corresponding inequality (1.1) to

$$
\left|\frac{1}{4}\left(f(a)+2 f\left(\frac{a+b}{2}\right)+f(b)\right)-\frac{1}{b-a} \int_{a}^{b} f(t) d t\right| \leq \frac{(b-a)^{2}}{48}\left\|f^{\prime \prime}\right\|_{\infty} .
$$

Until now, a large number of research papers and books have been written on Ostrowski inequalities and their numerous applications, see $([3,4,5,7$, $9,10,16,17,18,21,24,25,26])$.

On the other hand, the following inequality is well known in the literature as Simpson's inequality.

Theorem 3. Let $f:[a, b] \rightarrow \mathbf{R}$ be a four times continuously differentiable mapping on $(a, b)$ and $\left\|f^{(4)}\right\|_{\infty}=\sup \left|f^{(4)}(x)\right|<\infty$. Then, the following inequality holds:

$$
\left|\frac{1}{3}\left[\frac{f(a)+f(b)}{2}+2 f\left(\frac{a+b}{2}\right)\right]-\frac{1}{b-a} \int_{a}^{b} f(x) d x\right| \leq \frac{1}{2880}\left\|f^{(4)}\right\|_{\infty}(b-a)^{4} .
$$

Sarikaya et al. proved the following Simpson type inequality;

Theorem 4. [27] Let $f:[a ; b] \rightarrow \mathbf{R}$ is a different differentiable mapping on $I^{\circ}$ such that $f^{\prime} \in L_{1}[a, b]$, where $a, b \in I$ with $a<b$. If $\left|f^{\prime}\right|$ is a convex on $[a, b$,$] then the following inequality holds:$

$$
\left|\frac{1}{6}\left[f(a)+4 f\left(\frac{a+b}{2}\right)+f(b)\right]-\frac{1}{b-a} \int_{a}^{b} f(x) d x\right| \leq \frac{5(b-a)}{72}\left[\left|f^{\prime}(a)\right|+\left|f^{\prime}(b)\right|\right]
$$

For more papers on Simpson inequality, please refer to $([6,11,12,13,14$, $15,22,23,28,27,29,30,31,32,33,34])$.

\section{Multiplicative Calculus}

Recall multiplicative derivative which can be found in [8]. 
Definition 1. [8] Let $f: \mathbf{R} \rightarrow \mathbf{R}^{+}$be a positive function. The multiplicative derivative of the function $f$ is given by

$$
\frac{d^{*} f}{d t}(t)=f^{*}(t)=\lim _{h \rightarrow 0}\left(\frac{f(t+h)}{f(t)}\right)^{1 / h} .
$$

If $f$ has positive values and is differentiable at $t$, then $f^{*}$ exists and the relation between $f^{*}$ and ordinary derivative $f^{\prime}$ is as follows:

$$
f^{*}(t)=e^{[\log f(t)]^{\prime}}=e^{\frac{f^{\prime}(t)}{f(t)}} .
$$

If, additionally, the second derivative of $f$ at $t$ exists, then by an easy substitution, we obtain

$$
f^{* *}(t)=e^{\left[\log \circ f^{*}(t)\right]^{\prime}}=e^{[\log f(t)]^{\prime \prime}} .
$$

Here $(\ln f)^{\prime \prime}(t)$ exists because $f^{\prime \prime}(t)$ exist. Repeating this procedure $n$ times, we conclude that if $f$ is a positive function and its nth derivative at $t$ exists, then $f^{*(n)}(t)$ exists and

$$
f^{*(n)}(t)=e^{(\log f)^{(n)}(t)}, \quad n=1,2, \cdots .
$$

The following properties of multiplicative differentiable exist:

Theorem 1. [8] Let $f$ and $g$ be multiplicatively differentiable functions. If $c$ is arbitrary constant, then functions $c f, f g, f+g, f / g$ and $f^{g}$ are * differentiable and

(1) $(c f)^{*}(t)=f^{*}(t)$

(2) $(f g)^{*}(t)=f^{*}(t) g^{*}(t)$

(3) $(f+g)^{*}(t)=f^{*}(t)^{\frac{f(t)}{f(t)+g(t)}} g^{*}(t)^{\frac{g(t)}{f(t)+g(t)}}$

(4) $\left(\frac{f}{g}\right)^{*}(t)=\frac{f^{*}(t)}{g^{*}(t)}$

(5) $\left(f^{g}\right)^{*}(t)=f^{*}(t)^{g(t)} f(t)^{g^{\prime}(t)}$.

Recall also that the concept of the * integral called multiplicative integral is denoted by $\int_{a}^{b}(f(x))^{d x}$ which introduced by Bashirov et al. in [8]. While the sum of the terms of product is used in the definition of a classical Riemann integral of $f$ on $[a, b]$, the product of terms raised to power is used in the definition multiplicative integral of $f$ on $[a, b]$.

There is the following relation between Riemann integral and multiplicative integral: 
Proposition 1. [8] If $f$ is Riemann integrable on $[a, b]$, then $f$ is multiplicative integrable on $[a, b]$ and

$$
\int_{a}^{b}(f(x))^{d x}=\exp \left(\int_{a}^{b} \log (f(x)) d x\right) .
$$

Moreover, Bashirov et al. show that multiplicative integrable has the following results and properties:

Proposition 2. [8] If $f$ is positive and Riemann integrable on $[a, b]$, then $f$ is multiplicative integrable on $[a, b]$ and

(i) $\int_{a}^{b}\left((f(x))^{p}\right)^{d x}=\left(\int_{a}^{b}(f(x))^{d x}\right)^{p}$,

(ii) $\int_{a}^{b}(f(x) g(x))^{d x}=\int_{a}^{b}(f(x))^{d x} \cdot \int_{a}^{b}(g(x))^{d x}$,

(iii) $\int_{a}^{b}\left(\frac{f(x)}{g(x)}\right)^{d x}=\frac{\int_{a}^{b}(f(x))^{d x}}{\int_{a}^{b}(g(x))^{d x}}$,

(iv) $\int_{a}^{b}(f(x))^{d x}=\int_{a}^{c}(f(x))^{d x} \cdot \int_{c}^{b}(f(x))^{d x}, a \leq c \leq b$.

(v) $\int_{a}^{a}(f(x))^{d x}=1$ and $\int_{a}^{b}(f(x))^{d x}=\left(\int_{b}^{a}(f(x))^{d x}\right)^{-1}$.

Theorem 2 (Multiplicative Integration by Parts). [8] Let $f:[a, b] \rightarrow$ $\mathbf{R}$ be multiplicative differentiable, let $g:[a, b] \rightarrow \mathbf{R}$ be differentiable so the function $f^{g}$ is multiplicative integrable.

Then

$$
\int_{a}^{b}\left(f^{*}(x)^{g(x)}\right)^{d x}=\frac{f(b)^{g(b)}}{f(a)^{g(a)}} \cdot \frac{1}{\int_{a}^{b}\left(f(x)^{g^{\prime}(x)}\right) d x} .
$$

Theorem 3 (Multiplicative Taylor's Theorem for One Variable). [8] Let $A$ be an open interval and let $f: A \rightarrow \mathbf{R}$ be $n+1$ times ${ }^{*}$ differentiable on $A$. Then for any $x, x+h \in A$, there exists a number $\theta \in(0,1)$ such that

$$
f(x+h)=\prod_{k=1}^{n}\left(f^{*(k)}(x)\right)^{\frac{h^{k}}{k !}}\left(f^{*(n+1)}(x+\theta h)\right)^{\frac{h^{n+1}}{(n+1) !}} .
$$


For the our main results we need to following definition.

Definition 2. A non-empty set $K$ is said to be convex, if for every $a, b \in$ $K$ we have

$$
a+t(b-a) \in K, \forall t \in[0,1] .
$$

Definition 3. A function $f$ is said to be convex function on set $K$, if

$$
f(t x+(1-t) y) \leq t f(x)+(1-t) f(y), \forall t \in[0,1] .
$$

Definition 4. A function $f$ is said to be $\log$ or multiplicatively convex function on set $K$, if

$$
f(t x+(1-t) y) \leq[f(x)]^{t} \cdot[f(y)]^{1-t}, \forall t \in[0,1] .
$$

Hermite-Hadamard inequality for multiplicatively convex function is proved by Ali et al. as follows:

Theorem 4. [2] Let $f$ be a positive and multiplicatively convex function on interval $[a, b]$, then the following inequalities hold

$$
f\left(\frac{a+b}{2}\right) \leq\left(\int_{a}^{b}(f(x))^{d x}\right)^{\frac{1}{b-a}} \leq G(f(a), f(b)),
$$

where $G(.,$.$) is a geometric mean.$

For some more inequalities concerning (2.1), readers can read [1, 20].

\section{Ostrowski type inequalities for multiplicative integrals}

Before we prove our results, we give the following lemma.

Lemma 1. Let $f:[a, b] \rightarrow \mathbf{R}$ be multiplicative differentiable, let $g$ : $[a, b] \rightarrow \mathbf{R}$ and $h: J \subset \mathbf{R} \rightarrow[a, b]$ be two differentiable functions. Then we have

$$
\int_{a}^{b}\left(f^{*}(h(x))^{g(x) h^{\prime}(x)}\right)^{d x}=\frac{f(h(b))^{g(b)}}{f(h(a))^{g(a)}} \cdot \frac{1}{\left.\int_{a}^{b}(f(h(x)))^{g^{\prime}(x)}\right) d x} .
$$


Proof. The proof is obvious from the properties of multiplicative derivatives and integrals.

Lemma 2. Let $f: I^{0} \subseteq \mathbf{R} \rightarrow \mathbf{R}^{+}$be a multiplicative differentiable mapping on $I^{0}, a, b \in I^{0}$ with $a<b$. If $f^{*}$ is multiplicative integrable on $[a, b]$, then we have the following identity for multiplicative integrals

$$
f(x)\left(\int_{a}^{b}(f(t))^{d t}\right)^{\frac{1}{a-b}}=\int_{a}^{x}\left(\left[f^{*}(t)\right]^{\frac{t-a}{b-a}}\right)^{d t} \int_{x}^{b}\left(\left[f^{*}(t)\right]^{\frac{t-b}{b-a}}\right)^{d t} .
$$

Proof. Using the integration by parts for multiplicative integrals, we obtain

$$
\begin{aligned}
\int_{a}^{x}\left(\left[f^{*}(t)\right]^{\frac{t-a}{b-a}}\right)^{d t} \int_{x}^{b}\left(\left[f^{*}(t)\right]^{\frac{t-b}{b-a}}\right)^{d t} & =\frac{[f(x)]^{\frac{x-a}{b-a}}}{\int_{a}^{x}\left([f(t)]^{\frac{1}{b-a}}\right)^{d t}} \frac{1}{[f(x)]^{\frac{x-b}{b-a}}} \frac{1}{\int_{x}^{b}\left([f(t)]^{\frac{1}{b-a}}\right)^{d t}} \\
& =f(x)\left(\int_{a}^{b}(f(t))^{d t}\right)^{\frac{1}{a-b}} .
\end{aligned}
$$

Now, using Lemma 2, we give the following Theorems.

Theorem 1. Let $f: I^{0} \subset \mathbf{R} \rightarrow \mathbf{R}^{+}$be a multiplicative differentiable mapping on $I^{0}, a, b \in I^{0}$ with $a<b$. If $\left|\log f^{*}\right| \leq \log M$, then we have the following Ostrowski inequality for multiplicative integrals

$$
\left|f(x)\left(\int_{a}^{b}(f(t))^{d t}\right)^{\frac{1}{a-b}}\right| \leq M^{(b-a)\left[\frac{1}{4}+\frac{\left(x-\frac{a+b}{2}\right)^{2}}{(b-a)^{2}}\right]}
$$

for all $x \in[a, b]$.

Proof. Using Lemma 2, we obtain

$$
\left|f(x)\left(\int_{a}^{b}(f(t))^{d t}\right)^{\frac{1}{a-b}}\right|=\left|\int_{a}^{x}\left(\left[f^{*}(t)\right]^{\frac{t-a}{b-a}}\right)^{d t}\right|\left|\int_{x}^{b}\left(\left[f^{*}(t)\right]^{\frac{t-b}{b-a}}\right)^{d t}\right|
$$




$$
\begin{aligned}
& \leq \exp \left(\int_{a}^{x}\left|\log \left[f^{*}(t)\right]^{\frac{t-a}{b-a}}\right| d t\right) \exp \left(\int_{x}^{b}\left|\left[f^{*}(t)\right]^{\frac{t-b}{b-a}}\right| d t\right) \\
& =\exp \left(\int_{a}^{x}\left|\frac{t-a}{b-a} \log f^{*}(t)\right| d t\right) \exp \left(\int_{x}^{b}\left|\frac{t-b}{b-a} \log f^{*}(t)\right| d t\right) \\
& =\exp \left(\frac{1}{b-a} \int_{a}^{x}(t-a)\left|\log f^{*}(t)\right| d t\right) \exp \left(\frac{1}{b-a} \int_{x}^{b}(b-t)\left|\log f^{*}(t)\right| d t\right) .
\end{aligned}
$$

Since $\left|\log f^{*}\right| \leq \log M$, we get

$$
\begin{aligned}
& \left|f(x)\left(\int_{a}^{b}(f(t))^{d t}\right)^{\frac{1}{a-b}}\right| \leq \exp \left(\frac{\log M}{b-a} \int_{a}^{x}(t-a) d t+\frac{\log M}{b-a} \int_{x}^{b}(b-t) d t\right) \\
= & \exp \left(\frac{\log M}{b-a}\left[\frac{(x-a)^{2}}{2}+\frac{(b-x)^{2}}{2}\right]\right) \\
= & M^{(b-a)\left[\frac{1}{4}+\frac{\left(x-\frac{a+b}{2}\right)^{2}}{(b-a)^{2}}\right]} .
\end{aligned}
$$

Theorem 2. Let $f: I^{0} \subset \mathbf{R} \rightarrow \mathbf{R}^{+}$be a multiplicative differentiable mapping on $I^{0}, a, b \in I^{0}$ with $a<b$. If $f$ is increasing on $[a, b]$ and $f^{*}$ is multiplicatively convex on $[a, b]$, then we the following Ostrowski type inequality for multiplicative integrals

$\left|f(x)\left(\int_{a}^{b}(f(t))^{d t}\right)^{\frac{1}{a-b}}\right| \leq f^{*}(a)^{\left[\frac{(x-a)^{2}}{2(b-a)}+\frac{(b-x)^{3}-(x-a)^{3}}{3(b-a)^{2}}\right]} f^{*}(b)^{\left[\frac{(b-x)^{2}}{2(b-a)}+\frac{(x-a)^{3}-(b-x)^{3}}{3(b-a)^{2}}\right]}$

for all $x \in[a, b]$.

Proof. Using Lemma 2, we have 


$$
\begin{aligned}
& \left|f(x)\left(\int_{a}^{b}(f(t))^{d t}\right)^{\frac{1}{a-b}}\right| \\
= & \left|\int_{a}^{x}\left(\left[f^{*}(t)\right]^{\frac{t-a}{b-a}}\right)^{d t} \int_{x}^{b}\left(\left[f^{*}(t)\right]^{\frac{t-b}{b-a}}\right)^{d t}\right| \\
\leq & \exp \left(\frac{1}{b-a} \int_{a}^{x}(t-a) \log f^{*}(t) d t\right) \exp \left(\frac{1}{b-a} \int_{x}^{b}(b-t) \log f^{*}(t) d t\right) \\
= & \exp \left(\frac{1}{b-a} \int_{a}^{x}(t-a) \log f^{*}\left(\frac{b-t}{b-a} a+\frac{t-a}{b-a} b\right) d t\right) \\
& \times \exp \left(\frac{1}{b-a} \int_{x}^{b}(b-t) \log f^{*}\left(\frac{b-t}{b-a} a+\frac{t-a}{b-a} b\right) d t\right) .
\end{aligned}
$$

Since $f^{*}$ is multiplicatively convex, we obtain

$$
\begin{aligned}
& \left|f(x)\left(\int_{a}^{b}(f(t))^{d t}\right)^{\frac{1}{a-b}}\right| \\
\leq & \exp \left(\frac{1}{b-a} \int_{a}^{x}(t-a)\left[\frac{b-t}{b-a} \log f^{*}(a)+\frac{t-a}{b-a} \log f^{*}(b)\right] d t\right) \\
& \times \exp \left(\frac{1}{b-a} \int_{x}^{b}(b-t)\left[\frac{b-t}{b-a} \log f^{*}(a)+\frac{t-a}{b-a} \log f^{*}(b)\right] d t\right) \\
= & \exp \left(\frac{\log f^{*}(a)}{(b-a)^{2}} \int_{a}^{x}(t-a)(b-t) d t+\frac{\log f^{*}(b)}{(b-a)^{2}} \int_{a}^{x}(t-a)^{2} d t\right) \\
& \times \exp \left(\frac{\log f^{*}(a)}{(b-a)^{2}} \int_{x}^{b}(b-t)^{2} d t+\frac{\log f^{*}(b)}{(b-a)^{2}} \int_{x}^{b}(t-a)(b-t) d t\right),
\end{aligned}
$$

i.e., 


$$
\begin{aligned}
& \left|f(x)\left(\int_{a}^{b}(f(t))^{d t}\right)^{\frac{1}{a-b}}\right| \\
\leq & f^{*}(a)^{\frac{1}{(b-a)^{2}}\left[\frac{1}{2}(b-a)(x-a)^{2}-\frac{1}{3}(x-a)^{3}+\frac{1}{3}(b-x)^{3}\right]} f^{*}(b)^{\frac{1}{(b-a)^{2}}\left[\frac{1}{2}(b-a)(b-x)^{2}-\frac{1}{3}(b-x)^{3}+\frac{1}{3}(x-a)^{3}\right]} .
\end{aligned}
$$

Corollary 1. If we choose $x=\frac{a+b}{2}$ in Theorem 2, then we have the following Midpoint type inequalities for multiplicative integrals

$$
\left|f\left(\frac{a+b}{2}\right)\right| \leq\left[f^{*}(a) f^{*}(b)\right]^{\frac{b-a}{8}} .
$$

Now we give the following Ostrowski inequality for multiplicative integrals by using the Multiplicative Taylor's Theorem.

Theorem 3. Let $f: I^{0} \subset \mathbf{R} \rightarrow \mathbf{R}^{+}$be a multiplicative differentiable mapping on $I^{0}, a, b \in I^{0}$ with $a<b$. If $\left|\log f^{* *}\right| \leq \log M$, then we have the following Ostrowski inequality for multiplicative integrals

$$
\left|\sqrt{f(x)[f(a)]^{\frac{x-a}{b-a}}[f(b)]^{\frac{b-x}{b-a}}}\left(\int_{a}^{b}(f(t))^{d t}\right)^{\frac{1}{a-b}}\right| \leq M^{\frac{(b-a)^{2}}{4}\left[\frac{1}{12}+\frac{\left(x-\frac{a+b}{2}\right)^{2}}{(b-a)}\right]}
$$

for all $x \in[a, b]$. aki

Proof. By the Theorem 3, for $x, t \in(a, b)$ we have

$$
f(x)=f(t)\left[f^{*}(t)\right]^{x-t}\left[f^{* *}(\xi)\right]^{\frac{(x-t)^{2}}{2}}
$$

where $\xi=y+\theta(x-t)$. By the using multiplicative integral, we get 


$$
\begin{aligned}
{[f(x)]^{b-a} } & =\int_{a}^{b}\left(f(t)\left[f^{*}(t)\right]^{x-t}\left[f^{* *}(\xi)\right]^{\frac{(x-t)^{2}}{2}}\right)^{d t} \\
& =\int_{a}^{b}(f(t))^{d t} \cdot \int_{a}^{b}\left(\left[f^{*}(t)\right]^{x-t}\right)^{d t} \cdot \int_{a}^{b}\left(\left[f^{* *}(\xi)\right]^{\frac{(x-t)^{2}}{2}}\right)^{d t} .
\end{aligned}
$$

Here we have

(3.6) $\int_{a}^{b}\left(\left[f^{*}(t)\right]^{x-t}\right)^{d t}=\frac{[f(b)]^{x-b}}{[f(a)]^{x-a}} \cdot \frac{1}{\int_{a}^{b}\left([f(t)]^{-1}\right)^{d t}}=\frac{\int_{a}^{b}(f(t))^{d t}}{[f(a)]^{x-a}[f(b)]^{b-x}}$.

By the equalities (3.5) and (3.6), we have

$$
[f(x)]^{b-a}[f(a)]^{x-a}[f(b)]^{b-x}\left(\int_{a}^{b}(f(t))^{d t}\right)^{-2}=\int_{a}^{b}\left(\left[f^{* *}(\xi)\right]^{\frac{(x-t)^{2}}{2}}\right)^{d t} .
$$

Then, by using the assumption $\left|\log f^{* *}\right| \leq \log M$, it follows that

$$
\begin{aligned}
\left|[f(x)]^{b-a}[f(a)]^{x-a}[f(b)]^{b-x}\left(\int_{a}^{b}(f(t))^{d t}\right)^{-2}\right| & \leq \exp \left(\frac{1}{2} \int_{a}^{b}(x-t)^{2}\left|\log f^{* *}(\xi)\right| d t\right) \\
& \leq \exp \left(\frac{\log M}{2} \int_{a}^{b}(x-t)^{2} d t\right) \\
& =M^{\frac{(b-a)^{3}}{2}\left[\frac{1}{12}+\frac{\left(x-\frac{a+b}{2}\right)^{2}}{(b-a)}\right]} .
\end{aligned}
$$




\section{Simpson type inequalities for multiplicative integrals}

Lemma 3. Let $f: I^{0} \subseteq \mathbf{R} \rightarrow \mathbf{R}^{+}$be a multiplicative differentiable mapping on $I^{0}, a, b \in I^{0}$ with $a<b$. If $f^{*}$ is multiplicative integrable on $[a, b]$, then we have the following identity for multiplicative integrals

$$
\begin{aligned}
& \left(f(a) f(b)\left[f\left(\frac{a+b}{2}\right)\right]^{2}\right)^{\frac{1}{6}}\left(\int_{a}^{b}(f(x))^{d x}\right)^{\frac{1}{a-b}} \\
& =\left(\int_{0}^{1}\left(\left[f^{*}\left(\frac{1+t}{2} b+\frac{1-t}{2} a\right)\right]^{\left(\frac{t}{4}-\frac{1}{6}\right)}\right)^{d t}\right)^{(b-a)} \\
& \left(\int_{0}^{1}\left(\left[f^{*}\left(\frac{1+t}{2} a+\frac{1-t}{2} b\right)\right]^{\left(\frac{1}{6}-\frac{t}{4}\right)}\right)^{d t}\right)^{(b-a)} .
\end{aligned}
$$

Proof. From the Proposition 2 (i), we have

$$
\begin{aligned}
& \left(\int_{0}^{1}\left(\left[f^{*}\left(\frac{1+t}{2} b+\frac{1-t}{2} a\right)\right]^{\left(\frac{t}{4}-\frac{1}{6}\right)}\right) d t\right)^{(b-a)} \\
& \left(\int_{0}^{1}\left(\left[f^{*}\left(\frac{1+t}{2} a+\frac{1-t}{2} b\right)\right]^{\left(\frac{1}{6}-\frac{t}{4}\right)}\right) d t\right)^{(b-a)} \\
& =\int_{0}^{1}\left(\left[f^{*}\left(\frac{1+t}{2} b+\frac{1-t}{2} a\right)\right]^{\frac{b-a}{2}\left(\frac{t}{2}-\frac{1}{3}\right)}\right)^{d t} \\
& \int_{0}^{1}\left(\left[f^{*}\left(\frac{1+t}{2} a+\frac{1-t}{2} b\right)\right]^{\frac{b-a}{2}\left(\frac{1}{3}-\frac{t}{2}\right)}\right)^{d t}
\end{aligned}
$$

By using the Lemma 1, we obtain 


$$
\begin{aligned}
& \int_{0}^{1}\left(\left[f^{*}\left(\frac{1+t}{2} b+\frac{1-t}{2} a\right)\right]^{\frac{b-a}{2}\left(\frac{t}{2}-\frac{1}{3}\right)}\right)^{d t} \\
= & \frac{[f(b)]^{\frac{1}{6}}}{\left[f\left(\frac{a+b}{2}\right)\right]^{-\frac{1}{3}}} \frac{1}{\int_{0}^{1}\left(\left[f\left(\frac{1+t}{2} b+\frac{1-t}{2} a\right)\right]^{\frac{1}{2}}\right)^{d t}} \\
= & {\left[f\left(\frac{a+b}{2}\right)\right]^{\frac{1}{3}}[f(b)]^{\frac{1}{6}}\left[\int_{0}^{1}\left(f\left(\frac{1+t}{2} b+\frac{1-t}{2} a\right)\right)^{d t}\right]^{-\frac{1}{2}} } \\
= & {\left[f\left(\frac{a+b}{2}\right)\right]^{\frac{1}{3}}[f(b)]^{\frac{1}{6}}\left(\int_{\frac{a+b}{2}}^{b}(f(x))^{d x}\right)^{\frac{1}{a-b}} . }
\end{aligned}
$$

Similarly, we get

$$
\int_{0}^{1}\left(\left[f^{*}\left(\frac{1+t}{2} a+\frac{1-t}{2} b\right)\right]^{\frac{b-a}{2}\left(\frac{1}{3}-\frac{t}{2}\right)}\right)^{d t}=\left[f\left(\frac{a+b}{2}\right)\right]^{\frac{1}{3}}[f(b)]^{\frac{1}{6}}\left(\int_{a}^{\frac{a+b}{2}}(f(x))^{d x}\right)^{\frac{1}{a-b}} .
$$

By substituting the equalities (4.2) and (4.3) in (4.1), we have the required result (4.1).

Now, using Lemma 3, we give the following Theorems.

Theorem 1. Let $f: I^{0} \subset \mathbf{R} \rightarrow \mathbf{R}^{+}$be a multiplicative differentiable mapping on $I^{0}, a, b \in I^{0}$ with $a<b$. If $f$ is increasing on $[a, b]$ and $f^{*}$ is multiplicatively convex on $[a, b]$, then we have

(4.4) $\left|\left(f(a) f(b)\left[f\left(\frac{a+b}{2}\right)\right]^{2}\right)^{\frac{1}{6}}\left(\int_{a}^{b}(f(x))^{d x}\right)^{\frac{1}{a-b}}\right| \leq\left(f^{*}(a) f^{*}(b)\right)^{\frac{5(b-a)}{72}}$.

Proof. Using Lemma 3, we have

$$
\left|\left(f(a) f(b)\left[f\left(\frac{a+b}{2}\right)\right]^{2}\right)^{\frac{1}{6}}\left(\int_{a}^{b}(f(x))^{d x}\right)^{\frac{1}{a-b}}\right|
$$




$$
\begin{gathered}
\leq\left|\left[\int_{0}^{1}\left(\left[f^{*}\left(\frac{1+t}{2} b+\frac{1-t}{2} a\right)\right]^{\left(\frac{t}{4}-\frac{1}{6}\right)}\right)^{d t} \times \int_{0}^{1}\left(\left[f^{*}\left(\frac{1+t}{2} a+\frac{1-t}{2} b\right)\right]^{\left(\frac{1}{6}-\frac{t}{4}\right)}\right)^{d t}\right]^{b-a}\right| \\
\left.\leq|| \int_{0}^{1}\left(\left[f^{*}\left(\frac{1+t}{2} b+\frac{1-t}{2} a\right)\right]^{\left(\frac{t}{4}-\frac{1}{6}\right)}\right)^{d t}\left|\int_{0}^{1}\left(\left[f^{*}\left(\frac{1+t}{2} a+\frac{1-t}{2} b\right)\right]^{\left(\frac{1}{6}-\frac{t}{4}\right)}\right)^{d t}\right|\right]^{b-a} \\
\leq \exp \left((b-a) \int_{0}^{1}\left|\log \left[f^{*}\left(\frac{1+t}{2} b+\frac{1-t}{2} a\right)\right]^{\left(\frac{t}{4}-\frac{1}{6}\right)}\right| d t\right) \\
\times \exp \left((b-a) \int_{0}^{1}\left|\log \left[f^{*}\left(\frac{1+t}{2} a+\frac{1-t}{2} b\right)\right]^{\left(\frac{1}{6}-\frac{t}{4}\right)}\right| d t\right) \\
=\exp \left((b-a) \int_{0}^{1}\left|\frac{t}{4}-\frac{1}{6}\right| \log f^{*}\left(\frac{1+t}{2} b+\frac{1-t}{2} a\right) d t\right) \\
\times \exp \left((b-a) \int_{0}^{1}\left|\frac{1}{6}-\frac{t}{4}\right| \log f^{*}\left(\frac{1+t}{2} b+\frac{1-t}{2} a\right) d t\right) .
\end{gathered}
$$

By using the multiplicative convexity of $\left|f^{*}\right|$, it follows that

$$
\begin{aligned}
& \mid\left(f(a) f(b)\left[f\left(\frac{a+b}{2}\right)\right]^{2}\right)^{\frac{1}{6}}\left(\int_{a}^{b}(f(x))^{d x}\right)^{\frac{1}{a-b} \mid} \\
\leq & \exp \left((b-a) \int_{0}^{1}\left|\frac{t}{4}-\frac{1}{6}\right| \log \left(\left[f^{*}(a)\right]^{\left(\frac{1+t}{2}\right)}\left[f^{*}(b)\right]^{\left(\frac{1-t}{2}\right)}\right) d t\right) \\
\times & \left.\exp \left((b-a) \int_{0}^{1}\left|\frac{t}{4}-\frac{1}{6}\right| \log \left(\left[f^{*}(b)\right]\right]^{\left(\frac{1+t}{2}\right)}\left[f^{*}(a)\right]^{\left(\frac{1-t}{2}\right)}\right) d t\right) \\
= & \exp \left((b-a) \int_{0}^{1}\left|\frac{t}{4}-\frac{1}{6}\right|\left[\left(\frac{1+t}{2}\right) \log f^{*}(a)+\left(\frac{1-t}{2}\right) \log f^{*}(b)\right] d t\right) \\
\times & \exp \left((b-a) \int_{0}^{1}\left|\frac{t}{4}-\frac{1}{6}\right|\left[\left(\frac{1+t}{2}\right) \log f^{*}(b)+\left(\frac{1-t}{2}\right) \log f^{*}(a)\right] d t\right) \\
= & \left(f^{*}(a) f^{*}(b)\right)^{\frac{5(b-a)}{72}} .
\end{aligned}
$$


Example 1. Let $f:[0,1] \rightarrow \mathbf{R}^{+}, f(t)=e^{c t^{2}}$ with $c \in \mathbf{R}^{+}$. Then we have $f^{*}(t)=e^{2 c t}$. We also have $f(a)=f(0)=1, f(b)=f(1)=e^{c}$, $f\left(\frac{a+b}{2}\right)=f\left(\frac{1}{2}\right)=e^{\frac{c}{4}}, f^{*}(a)=f^{*}(0)=1$ and $f^{*}(b)=f^{*}(1)=e^{2 c}$. On the other hand

$$
\left(\int_{a}^{b}(f(x))^{d x}\right)^{\frac{1}{b-a}}=\int_{0}^{1}\left(e^{c x^{2}}\right)^{d x}=e^{\frac{c}{3}} .
$$

Then it follows that

$$
\left|\left(f(a) f(b)\left[f\left(\frac{a+b}{2}\right)\right]^{2}\right)^{\frac{1}{6}}\left(\int_{a}^{b}(f(x))^{d x}\right)^{\frac{1}{a-b}}\right|=e^{-\frac{c}{12}}
$$

and

$$
\left(f^{*}(a) f^{*}(b)\right)^{\frac{5(b-a)}{72}}=\left(e^{2 c}\right)^{\frac{5}{72}}=e^{\frac{5 c}{36}}
$$

\section{Applications to Special Means of Real Numbers}

We will consider the following means for real numbers $a, b$ with $a \neq b$. We have

(1) The arithmetic means for $a, b \in \mathbf{R}^{+}$:

$\mathrm{A}:=\mathrm{A}(a, b)=\frac{a+b}{2}$.

(2) The geometric means for $a, b \in \mathbf{R}^{+}$:

$\mathrm{G}:=\mathrm{G}(a, b)=\sqrt{a b}$.

(3) The harmonic means for $a, b \in \mathbf{R}^{+} \backslash\{0\}$ :

$\mathrm{H}:=\mathrm{H}(a, b)=\frac{2 a b}{a+b}$.

(4) The logarithmic means for $a, b \in \mathbf{R}^{+}$and $|a| \neq|b|$ :

$\mathrm{L}:=\mathrm{L}(a, b)=\frac{b-a}{\ln |b|-\ln |a|}$.

(5) The identric means for $a, b \in \mathbf{R}^{+} \backslash\{0\}$ :

$$
I=I(a, b):=\left\{\begin{array}{cc}
\frac{1}{e}\left(\frac{b^{b}}{a^{a}}\right)^{\frac{1}{b-a}}, & b \neq a \\
a, & b=a
\end{array} .\right.
$$

(6) The generalized logarithmic means for $a, b \in \mathbf{R} \backslash\{0\}$ and $p \in \mathbf{R} \backslash\{-1,0\}$ : $\mathrm{L}_{p}:=L_{p}(a, b)=\left[\frac{b^{p+1}-a^{p+1}}{(p+1)(b-a)}\right]^{\frac{1}{n}}$. 
It is well-known that $L_{-1}=L$ and $L_{0}=I$, because $L_{p}$ is monotonic nondecreasing over $p \in \mathbf{R}$.

Now, using our main results we give some applications to special means of real numbers. Some error estimates for the special means are obtain below.

(1) Consider a positive function $f:[a, b] \rightarrow \mathbf{R}$ with $b>a>0$. If $f(x)=e^{x^{p}}$ with $p>1$, then we have

$$
\left(\int_{a}^{b} f(x)^{d x}\right)^{\frac{1}{b-a}}=e^{L_{p}^{p}(a, b)}
$$

(a) From inequality (3.1), we have following relation of means for $M=$ $\exp \left(p b^{p-1}\right)$

$$
e^{x^{p}-L_{p}^{p}} \leq M^{(b-a)\left[\frac{1}{4}+\frac{x-A}{(b-a)^{2}}\right]} .
$$

For a moment, if we put

(i) $x=A$, then we have

$$
e^{A^{p}-L_{p}^{p}} \leq M^{\frac{(b-a)}{4}} .
$$

(ii) $x=G$, then we get

$$
e^{G^{p}-L_{p}^{p}} \leq M^{(b-a)\left[\frac{1}{4}+\frac{G-A}{(b-a)^{2}}\right]} .
$$

(iii) One can write similar inequalities by choosing $x=H, L, I$.

(b) From inequality (3.4), we have following relation for means with $M=$ $\exp \left(p(p-1) b^{p-2}\right)$

$$
\left(e^{x^{p}+a^{p}\left(\frac{x-a}{b-a}\right)+b^{p}\left(\frac{b-x}{b-a}\right)-2 L_{p}^{p}(a, b)}\right)^{\frac{1}{2}} \leq M^{(b-a)\left[\frac{1}{12}+\frac{\left(x-\frac{a+b}{2}\right)}{(b-a)^{2}}\right] .}
$$

If we choose following assumptions, then we have

(i) $x=A$, we have

$$
\left(e^{A^{p}+A\left(a^{p}, b^{p}\right)-2 L_{p}^{p}}\right)^{\frac{1}{2}} \leq M^{\frac{(b-a)}{12}} .
$$

(ii) $x=G$, we get

$$
\left(e^{G^{p}+a^{p}\left(\frac{G-a}{b-a}\right)+b^{p}\left(\frac{b-G}{b-a}\right)-2 L_{p}^{p}(a, b)}\right)^{\frac{1}{2}} \leq M^{(b-a)\left[\frac{1}{12}+\frac{\left(G-\frac{a+b}{2}\right)}{(b-a)^{2}}\right]} .
$$


(iii) Similar inequalities can be written for $x=H, L, I$.

(2) Consider a positive function $f:[a, b] \rightarrow \mathbf{R}$ with $b>a>0$. If $f(x)=$ $e^{\frac{1}{p+1} x^{p+1}}$ with $p \geq 1$, then

$$
f^{*}(x)=e^{x^{p}}
$$

is multiplicatively convex on given domain and

$$
\left(\int_{a}^{b} f(x)^{d x}\right)^{\frac{1}{b-a}}=e^{\frac{1}{p+1} L_{p+1}^{p+1}(a, b)} .
$$

From inequality (3.2), we have

$$
\begin{aligned}
& e^{x^{p+1}-L_{p+1}^{p+1}(a, b)} \\
\leq & e^{a^{p}\left[\frac{(x-a)^{2}}{2(b-a)}+\frac{(b-x)^{3}-(x-a)^{3}}{3(b-a)^{2}}\right]+b^{p}\left[\frac{(b-x)^{2}}{2(b-a)}+\frac{(x-a)^{3}-(b-x)^{3}}{3(b-a)^{2}}\right]} .
\end{aligned}
$$

For an instance, if we choose

(i) $x=A$ or from inequality (3.3), we have

$$
e^{A^{p+1}-L_{p+1}^{p+1}(a, b)} \leq\left(e^{A\left(a^{p}, b^{p}\right)}\right)^{\frac{b-a}{4}} .
$$

(ii) $x=G$, we get

$$
\begin{aligned}
& e^{G^{p+1}-L_{p+1}^{p+1}(a, b)} \\
\leq & e^{a^{p}\left[\frac{(G-a)^{2}}{2(b-a)}+\frac{(b-G)^{3}-(G-a)^{3}}{3(b-a)^{2}}\right]+b^{p}\left[\frac{(b-G)^{2}}{2(b-a)}+\frac{(G-a)^{3}-(b-G)^{3}}{3(b-a)^{2}}\right]} .
\end{aligned}
$$

(iii) Similar inequalities can be written for $x=H, L, I$.

(3) Consider a positive function $f:[a, b] \rightarrow \mathbf{R}$ with $b>a>0$. If $f(x)=$ $e^{\frac{1}{p+1} x^{p+1}}$ with $p \geq 1$, then

$$
f^{*}(x)=e^{x^{p}}
$$

is multiplicatively convex on given domain and

$$
\left(\int_{a}^{b} f(x)^{d x}\right)^{\frac{1}{b-a}}=e^{\frac{1}{p+1} L_{p+1}^{p+1}(a, b)} .
$$

From inequality (4.4), we have

$$
\left(e^{A\left(a^{p+1}, b^{p+1}\right)+A^{p+1}-3 L_{p+1}^{p+1}(a, b)}\right)^{\frac{1}{3(p+1)}} \leq\left(e^{A\left(a^{p}, b^{p}\right)}\right)^{\frac{5(b-a)}{36}} .
$$




\section{Conclusion}

In this work, authors established Ostrowski and Simpson type inequalities in the setting of multiplicative calculus. We also gave applications to special means of real numbers by utilizing our newly established results. The results in this paper can be very crucial in the field of integral inequalities and multiplicative calculus. Interested readers can establish several new results in this field by using our new idea.

\section{Conflict of Interest}

The authors declare that they have no conflict of interests.

\section{Acknowledgment}

This work is partially supported by the National Natural Science Foundation of China (No.11971241).

\section{References}

[1] M. A. Ali, M. Abbas, H. Budak, and A. Kashuri, "Some new HermiteHadamard inequalities in multiplicative calculus", TWMS Journal of applied and engineering mathematics, Accepted, 2020.

[2] M. A. Ali, M. Abbas, Z. Zhang, I. B. Sial, and R. Arif, "On integral inequalities for product and quotient of two multiplicatively convex functions", Asian research journal of mathematics, vol. 12, no. 3, Art ID. 47297, 2019, doi: 10.9734/ arjom/2019/ v12i330084

[3] M. W. Alomari, M. Darus, S. S. Dragomir, and P. Cerone, "Ostrowski type inequalities for functions whose derivatives are s-convex in the second sense", Applied mathematics letters, vol. 23, no. 9, pp. 1071-1076, 2010, doi: 10.1016/ j.aml.2010.04.038

[4] M. W. Alomari, M. E. Özdemir, and H. Kavurmac, "On companion of Ostrowski inequality for mappings whose first derivatives absolute value are convex with applications", Miskolc mathematical notes, vol. 13, no. 2, pp. 233-248, 2012, doi: 10.18514/ MMN.2012.480 
[5] M. W. Alomari and M. Darus, "Some Ostrowski's type inequalities for convex functions with application", RGMIA research report collection, vol. 13, no. 1, Art ID. 3, 2010. [On line]. Available: https:// bit.ly/ 2Rm15a7

[6] M. W. Alomari, M. Darus, and S. S. Dragomir, "New inequalities of Simpson's type for sconvex functions with applications", RGMIA research report collection, vol. 12, no. 4, Art ID. 9, 2009. [On line]. Available: https:/ / bit.ly/ 33Pdtlw

[7] N. S. Barnett and S. S. Dragomir, "An Ostrowski type inequality for double integrals and applications for cubature formulae", Soochow journal of mathematics, vol. 27, no. 1, pp. 109-114, 2001. [On line]. Available: https:/ / bit.ly/ 3ffBwQ0

[8] A. E. Bashirov, E. M. Kurpınar, and A. Özyapıcl, "Multiplicative calculus and its applications", Journal of mathematical analysis and applications, vol. 337, no. 1, pp. 36-48, 2008, doi: 10.1016/j.jmaa.2007.03.081

[9] P. Cerone and S. S. Dragomir, "Ostrowski type inequalities for functions whose derivatives satisfy certain convexity assumptions", Demonstratio mathatica, vol. 37, no. 2, pp. 299-308, 2004, doi: 10.1515/ dema-2004-0208

[10] S. S. Dragomir,"Ostrowski type inequalities for functions whose derivatives are h-convex in absolute value", Tbilisi mathematical journal, vol. 7, no. 1, pp. 1-17, 2014, doi: 10.2478/ tmj-2014-0001

[11] S. S. Dragomir, R. P. Agarwal, and P. Cerone, "On Simpson's inequality and applications", Journal of inequalities and applications, vol. 5, no. 6, pp. 533-579, 2000. [On line]. Available: https:/ / bit.ly/ 33NjSh6

[12] S. S. Dragomir, “On Simpson's quadrature formula for differentiable mappings whose derivatives belong to lp spaces and applications", Journal of Korean Society for Industrial and Applied Mathematics, vol. 2, no. 2, pp. 57-65, 1998. [On line]. Available: https:/ / bit.ly/ 3uTuWVT

[13] S. S. Dragomir, "On Simpson's quadrature formula for Lipschitzian mappings and applications", Soochow journal of mathematics, vol. 25, pp. 175-180, 1999.

[14] S. Hussain and S. Qaisar, "More results on Simpson's type inequality through convexity for twice differentiable continuous mappings", SpringerPlus, vol. 5, Art ID. 77, 2016, doi: 10.1186/ s40064-016-1683-x

[15] Z. Liu, "An inequality of Simpson type", Proceedings - Royal Society. Mathematical, physical and engineering sciences, vol. 461, no. 2059, pp. 2155-2158, 2005, doi: 10.1098/ rspa.2005.1505 
[16] G. V. Milovanović, "On some integral inequalities”, Publikacije Elektrotehničkog fakulteta Univerziteta u Beogradu. Serija Matematika i fizika, vol. 498, no. 541, pp. 119-124, 1975. [On line]. Available: https:/ / bit.ly/ 3eURyjv

[17] G. V. Milovanović and J. E. Pečarić, "On generalization of the inequality of A. Ostrowski and some related applications", Publikacije Elektrotehničkog fakulteta Univerziteta u Beogradu. Serija Matematika i fizika, vol. 544, no. 576, pp. 155-158, 1976. [On line]. Available: https:/ / bit.ly/ 3fkZNUS

[18] M. A. Noor and M. U. Awan, "Some integral inequalities for two kinds of convexities via fractional integrals", Transylvanian journal of mathematics and mechanics, vol. 5, no. 2, pp. 129-136, 2013. [On line]. Available: https:/ / bit.ly/ 3uRGSrj

[19] A. M. Ostrowski, “Über die absolutabweichung einer differentiebaren funktion von ihrem integralmitelwert", Commentarii Mathematici Helvetici, vol. 10, pp. 226-227, 1938. [On line]. Available: https:/ / bit.ly/ 3w808iB

[20] S. Özcan, "Some integral inequalities of Hermite-Hadamard type for multiplicatively preinvex functions", AIMS mathematics, vol. 5, no. 22, pp. 1505-1518, 2020, doi: 10.3934/ math.2020103

[21] M. E. Özdemir, H. Kavurmacl, and M. Avcl, "Ostrowski type inequalities for convex functions", Tamkang journal of mathematics, vol. 45, no. 4, pp. 335-340, 2014, doi: 10.5556/j.tkjm. 45.2014 .1143

[22] M. E. Özdemir, A. O. Akdemir, and H. Kavurmacl, "On the Simpson's inequality for convex functions on the co-ordinates", Turkish journal of analysis and number theory, vol. 2, no. 5, pp. 165-169, 2014, doi: $10.12691 /$ tjant-2-5-2

[23] J. Pecaric and S. Varosanec, "A note on Simpson's inequality for functions of bounded variation", Tamkang journal of mathematics, vol. 31, no. 3, pp. 239-242, 2000, doi: 10.5556/j.tkjm.31.2000.398

[24] M. Z. Sarikaya, "On the Ostrowski type integral inequality", Acta Mathematica Universitatis Comenianae, vol. 79, no. 1, pp. 129-134, 2010. [On line]. Available: https:/ / bit.ly/ 3hs60B5

[25] M. Z. Sarikaya, "On the Ostrowski type integral inequality for double integrals", Demonstratio mathematica, vol. 45, no. 3, 2012, doi: 10.1515/ dema-2013-0398

[26] M. Z. Sarikaya and H. Ogunmez, "On the weighted Ostrowski type integral inequality for double integrals", Arabian journal for science and engineering, vol. 36, no. 6, pp. 1153-1160, 2011, doi: 10.1007/s13369-011-0102-4 
[27] M. Z. Sarikaya, E. Set and M. E. Ozdemir, "On new inequalities of Simpson's type for convex functions", RGMIA research report collection, vol. 13, no. 2, 2010, Article2. [On line]. Available: https:/ / bit.ly/ 3woiUV5

[28] M. Z. Sarikaya, E. Set, M. E. Özdemir, “On new inequalities of Simpson's type for s-convex functions", Computers and mathematics with applications, vol. 60, no. 8, pp. 2191-2199, 2010, doi:10.1016/j.camwa. 2010.07.033

[29] M. Z. Sarikaya, E. Set, and M. E. Özdemir, "On new inequalities of Simpson's type for functions whose second derivatives absolute values are convex", Journal of applied mathematics, statistics and informatics, vol. 9, no. 1, 2013. [On line]. Available: https:/ / bit.ly/ 33ODEck

[30] M. Z. Sarikaya, T. Tunç, and H. Budak, "Simpson's type inequality for Fconvex function", Facta Universitatis. Series mathematics and informatics, vol. 32, no. 5, 2017, doi: 10.22190/ FUMI1705747S

[31] E. Set, M. E. Özdemir, and M. Z. Sarikaya, "On new inequalities of Simpson's type for quasi-convex functions with applications", Tamkang journal of mathematics, vol. 43, no. 3, pp. 357-364, 2012, doi: 10.5556/ j.tkjm.43.2012.357-364

[32] E. Set, M. Z. Sarikaya, and N. Uygun, “On new inequalities of Simpson's type for generalized quasi-convex functions", Advances in inequalities and applications, vol. 2017, Art ID. 3, 2017. [On line]. Available: https:/ / bit.ly/ 3uViHYV

[33] K. L. Tseng, G. S. Yang, and S.S. Dragomir, "On weighted Simpson type inequalities and applications", Journal of mathematical inequalities, vol. 1, no. 1, pp. 13-22, 2007, doi: 10.7153/jmi-01-02

[34] N. Ujevicć, "Double integral inequalities of Simpson type and applications", Journal of applied mathematics and computations, vol. 14, no. 1-2, pp. 213-223, 2004, doi: 10.1007/ BF02936109 